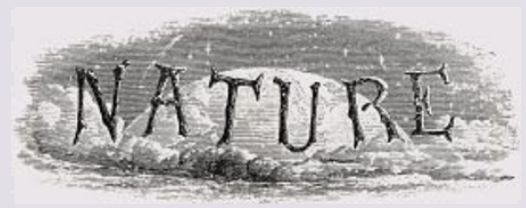

100 YEARS AGO

The action of magnetism on the propagation of light in a transparent medium has been rightly regarded as one of the most beautiful of Faraday's great scientific discoveries. Like most important discoveries it was no result of accidental observation, but was the outcome of long and patient inquiry. Guided by a conviction that (to quote his own words) "the various forms under which forces of matter are made manifest have one common origin," he made many attempts to discover a relation between light and electricity, but for very long with negative results. Still, however, retaining a strong persuasion that his view was correct, and that some such relation must exist, he was undiscouraged, and only proceeded to search for it more strictly and carefully than ever. At last, as he himself says, he "succeeded in magnetising and electrifying a ray of light, and in illuminating a magnetic line of force."

From Nature 17 August 1899

50 YEARS AGO

Osborne Reynolds, in a short paper entitled "On the Action of Rain to Calm the Sea", discussed the action of rain in calming a rough sea. He expressed the opinion that each drop of rain produces a vortex ring which, on descending into the water, transfers momentum from the surface layers to the underneath layers, thus reducing the relative motion of the layers. It was suggested to us by Prof. A. H. Gibson that further experiments were desirable to confirm the above theory. It has now been found that whereas vortex rings are, in fact, produced when the height of dropping, and hence the velocity of the drop, is small, only 'splash and surface effects' are produced when the height is great. ... It may be concluded, therefore, that very few, if any, of the drops in a normal rain-storm actually produce vortex rings when they strike the surface of water. Rather is the kinetic energy of the drop dissipated in shock and splash at the surface, and thus the sphere of influence of the drop is not as great as suggested by Reynolds.

From Nature 20 August 1949. rings now appear to be common features of the giant planets, their expected lifetimes are far shorter than the age of the Solar System. Rings should eventually collapse because of gravitational interactions with nearby moons, and the moons themselves should be destroyed by meteoroid impacts. The emerging view is that the ring-moon systems are not ancient structures existing since the Solar System began, but transient phenomena in which the death of a moon is also the birth of a new ring. If this is so, then the stable ring arcs of Neptune are of considerable interest as an intermediate structure between accreted moons and distributed rings. Perhaps we are seeing the cloud of debris from a disrupted moon, whose expansion was arrested by its chance proximity to a family of resonances capable of confining it.

Despite these puzzles, rings remain useful as dynamical laboratories in which astronomers can observe the processes that work in much larger astrophysical systems, such as galaxies and protoplanetary disks. On one level, the new reports are noteworthy simply as illustrations of how far astronomical methods have improved in the past decade, enabling two teams to detect a tiny feature a fraction away (less than two arc seconds) from a planet that is $10^{6}$ times brighter. The ability to image such features directly will reduce the need for astronomers to wait patiently for the occasional bright star to pass behind the rings (although stellar occultations will always remain useful because of the extraordinary geometric precision they provide). Even though the interactions between Neptune's moons and ring arcs just became a great deal murkier, the good news is that the dynamics of some of the Solar System's oddest structures are becoming much easier to study.

Mark R. Showalter is in the Space

Telecommunications and Radioscience Laboratory, Stanford University, Stanford, California 94305, USA. e-mail:showalter@ringside.arc.nasa.gov

1. Hubbard, W. B. et al. Nature 319, 636-640 (1986)

2. Sicardy, B. et al. Nature 400, 731-733 (1999).

3. Dumas, C., Terrile, R. J., Smith, B. A., Schneider, G. \& Becklin, E. E. Nature 400, 733-735 (1999).

4. Elliot, J. L., Dunham, E. W. \& Mink, D. J. Nature 267, 328-330 (1977).

Goldreich, P. \& Tremaine, S. Nature 277, 97-99 (1979).

6. Goldreich, P., Tremaine, S. \& Borderies, N. Astron. J. 92 , 490-494 (1986).

Lissauer, J. J. Nature 318, 544-545 (1985).

8. Porco, C. C. Science 253, 995-1001 (1991)

9. Sicardy, B. \& Lissauer, J. J. Adv. Space Res. 12, 1181-1195 (1992).

\section{Tripping the light fantastic}

Harry Smith

$\square$ hotosynthesis fuels life as we know it, so we should all be grateful that evolution has provided plants with sensory mechanisms that allow them to adapt precisely to sunlight. These signal-transducing photoreceptors tell the plant about the intensity, spectral quality, direction and periodic timing of solar radiation, setting off metabolic and developmental changes that optimize the photosynthetic absorption of photons. The most intensively researched photoreceptors are the five members of the phytochrome family (known as phytochromes A to E in the tiny weed Arabidopsis). Phytochromes are photochromic pigments - that is, they are photochemically converted from the biologically inactive $\mathrm{Pr}$ form to the active Pfr form by absorbing red photons. Conversely, Pfr can be converted back to Pr by absorbing far-red photons.

For more than 40 years, the Holy Grail of phytochrome research has been the molecular mechanism of Pfr action. Ni et al. ${ }^{1}$ now provide compelling data (on page 781 of this issue) about an interaction between phytochrome $\mathrm{B}$ and its putative reaction partner, phytochrome-interacting factor-3 (PIF3). The authors also speculate on a short-cut mechanism that links phytochrome photoconversion to gene regulation. This work is the latest stage in a sustained attack on the mechanism of phytochrome action from the laboratory of Peter Quail. And it is the first demonstration of photoreversible binding of a phytochrome to its putative signalling partner in vitro.

Quail's laboratory had already identified PIF3 by two separate approaches. In the first, Ni et al. ${ }^{2}$ used a yeast two-hybrid screen to fish for factors that interact with nonphotoactive carboxy-terminal fragments of phytochrome A and phytochrome B. (Phytochrome molecules are thought to act, at least in part, through a region in the carboxy terminus.) $\mathrm{Ni}$ and colleagues found ${ }^{2}$ that PIF3 binds to native phytochrome A and phytochrome B. Overexpression of PIF3 in the sense orientation increased light sensitivity; overexpression in the antisense orientation strongly decreased it. Crucially, binding of PIF3 was reduced with a series of phytochrome A and phytochrome B molecules carrying missense mutations that caused loss of in vivo activity. In the second approach, Halliday et al. ${ }^{3}$ isolated a new mutant of Arabidopsis with enhanced sensitivity to red light. The mutation turned out to be an insertion in the promoter of the PIF3 gene, causing the gene to be overexpressed. This study provided further evidence that PIF3 is involved invivo, and confirmed that it positively regulates phytochrome action.

Perhaps the most intriguing features of PIF3 are its nuclear localization and 
characterization as a basic helix-loop-helix protein with putative transcription-factor activity. Phytochrome action has long been thought to involve the selective regulation of gene expression, and there is evidence that the phytochrome translocates into the nucleus after conversion to $\mathrm{Pfr}^{4}$. Ni et al. ${ }^{1}$ now show the photoreversible interaction of phytochrome $\mathrm{B}$ with PIF3 in vitro. The dynamic characteristics of this interaction fit the photobiological in vivo information. The authors show that PIF3 binds to phytochrome B only after the phytochrome has been photoconverted from $\operatorname{Pr}_{B}$ to $\operatorname{Pfr}_{B}$. Reconversion of $\mathrm{Pfr}_{\mathrm{B}}$ to $\operatorname{Pr}_{\mathrm{B}}$ by far-red light abruptly terminates the association. The binding of PIF3 to $\mathrm{Pfr}_{\mathrm{B}}$ seems rather slow compared with in vivo response kinetics. However, the binding was done at $4{ }^{\circ} \mathrm{C}$, and dissociation on treatment with far-red light was reassuringly rapid.

As in previous experiments, missense mutations in phytochrome B caused sub- stantial reductions in binding to PIF3. Although details of the binding process still need to be worked out (for example, stoichiometry data are not yet available), binding sites in both the amino- and carboxy-terminal domains are essential for full association. We already know that photoisomerization of the light-absorbing moiety (chromophore) causes conformational changes in the holoprotein ${ }^{5}$. Now, the data of Ni et al. ${ }^{1}$ support a simple hypothesis involving three steps: translocation of $\mathrm{Pfr}_{\mathrm{B}}$ to the nucleus; binding of $\mathrm{Pfr}_{\mathrm{B}}$ to the constitutively nuclear PIF3; and interaction of the $\mathrm{Pfr}_{\mathrm{B}}$-PIF3 complex - either directly or indirectly — with target genes.

The apparent simplicity of this idea may, of course, turn out to be illusory. Other factors bind to phytochromes (including the cryptochrome photoreceptor CRY1) ${ }^{6}$. Indeed, one such factor (phytochrome kinase substrate-1, PKS1), fished out by the two-hybrid screen, is a substrate for

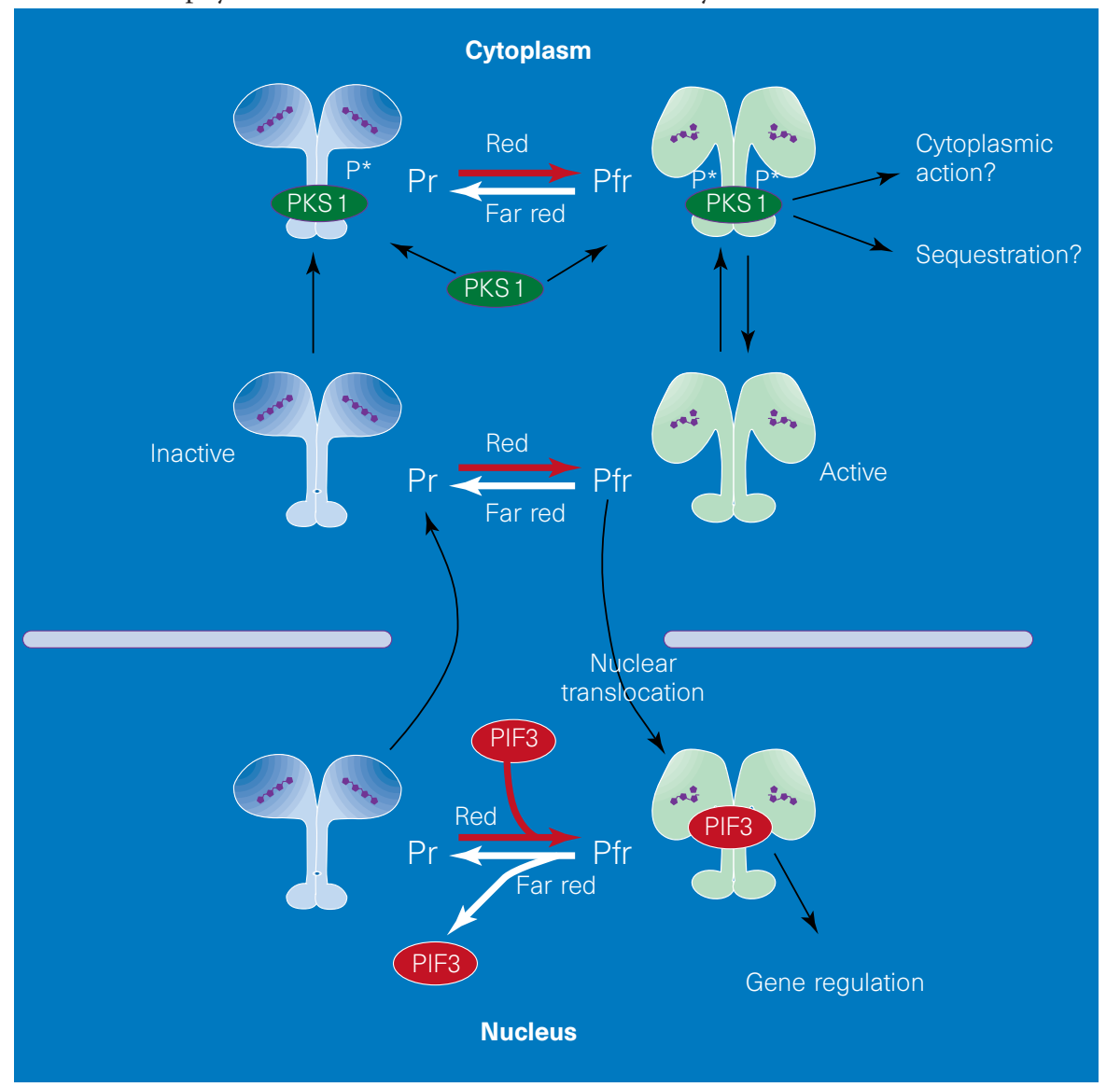

Figure 1 Main steps in phytochrome action. Absorption of a red photon by the inactive Pr form of a phytochrome causes a conformational change in the dimeric photoreceptor molecule. In the Pfr form, the phytochrome translocates to the nucleus where it binds to a putative reaction partner, PIF3, which is constitutively found in the nucleus and has the characteristics of a basic helix-loop-helix transcription factor. The Pfr-PIF3 complex initiates gene regulation, either directly or through unknown intermediates. Reversion of Pfr to Pr by far-red light results in rapid dissociation of PIF3, interrupting signal transduction. In the Pr form, the phytochrome slowly relocates to the cytoplasm. Here, in either the Pr or Pfr form, it can bind to a kinase substrate (PKS1), which may be involved in retention of the phytochrome in the cytoplasm or in its release for translocation. Phosphorylation of PKS1 is enhanced with Pfr, and may be the prelude to cytoplasmic action. So, several steps are susceptible to regulation by absorption of light by the photoreceptor: phosphorylation, nuclear translocation, association with PIF3 and transfer of signal transduction to PIF3. phytochrome A-mediated serine/threonine kinase activity ${ }^{7}$. There has been much controversy about whether the phytochromes are kinases ${ }^{8}$. But the accumulated evidence, including homologies with phytochromelike molecules in the cyanobacteria ${ }^{9,10}$, is so strong that the question now is the extent to which their kinase properties are implicated in signal transduction.

Earlier this year Fankhauser et al. ${ }^{7}$ showed that the cytoplasmically located PKS1 binds to, and is phosphorylated by, both $\operatorname{Pr}_{\mathrm{A}}$ and $\mathrm{Pfr}_{\mathrm{A}}$, but that phosphorylation activity is between 2- and 2.5-fold higher with the Pfr form. If this were to initiate a kinase cascade, it could be enough to explain the huge signal amplification observed in vivo. But these authors propose an intriguing alternative. Based on analogy with activation of NF- $\kappa$ B in mammalian cells ${ }^{11}$, they suggest that binding to PKS1 may be important for retaining phytochrome $\mathrm{A}$ in the cytoplasm. In this way, translocation of the phytochrome to the nucleus would be negatively regulated. Certainly, PKS1 negatively regulates the action of phytochrome A, as judged from overexpression experiments ${ }^{7}$. So, PKS1 may be a cytoplasmic anchoring protein, and phytochrome-mediated phosphorylation of PKS1 might release phytochrome A to move into the nucleus.

The differences between the results of $\mathrm{Ni}$ et al. ${ }^{1}$ and Fankhauser et al. ${ }^{7}$ may lie in their choice of phytochrome A or phytochrome B as the experimental material, although PIF3 and PKS1 bind to both. It is premature to try to resolve all the questions about phytochrome action, and Fig. 1 shows only one of several possibilities. Such a scheme could account for phytochrome-mediated responses that operate through gene regulation, or through other actions - rapid alterations in growth rate or membrane properties, or chloroplast relocation - that probably emanate from a separate primary action located in the cytoplasm.

The transition between molecular genetics and biochemistry is never easy, and major caveats need to be mentioned. For example, mutants provide evidence for both common and separate pathways of phytochrome A and phytochrome B action. Furthermore, pharmacological evidence points towards a number of intermediary metabolites (such as guanine-nucleotide-binding (G) proteins, $\mathrm{Ca}^{2+}$ and cyclic GMP) in signal transduction. Integrating all these potential branches into a single scheme will test our ingenuity. The first steps in the choreography of phytochrome signal transduction are beginning to be worked out - but will the dance be a pas de deux or a hoedown?

Harry Smith is in the Department of Biology, University of Leicester, Leicester LE1 7RH, UK. e-mail:has@leicester.ac.uk

1. Ni, M., Tepperman, J. M. \& Quail, P. H. Nature 400, 781-784 (1999). 
2. Ni, M., Tepperman, J. M. \& Quail, P. H. Cell 95, 657-667 (1998).

3. Halliday, K. J., Hudson, M., Ni, M., Qin, M. \& Quail, P. H.

Proc. Natl Acad. Sci. USA 96, 5832-5837 (1999).

4. Yamaguchi, R., Nakamura, M., Mochizuki, N., Kay, S. A. \& Nagatani, A. J. Cell Biol. 145, 437-445 (1999).

5. Song, P.-S. J. Biochem. Mol. Biol. 32, 215-225 (1999).

6. Ahmad, M., Jarillo, J. A., Smirnova, O. \& Cashmore, A. R. Mol. Cell 1, 939-948 (1998).
7. Fankhauser, C. et al. Science 284, 1539-1541 (1999)

8. Cashmore, A. R. Proc. Natl Acad. Sci. USA 95, 13358-13360 (1998)

9. Kehoe, D. M. \& Grossman, A. R. Science 273, 1409-1412 (1996).

10. Yeh, K. C., Wu, S. H., Murphy, J. P. \& Lagarias, J. C. Science 277, 1505-1508 (1997).

11. Stancovski, I. \& Baltimore, D. Cell 91, 299-302 (1997)

\section{Atmospherio chemistry}

\section{Sulphur emissions from ships}

\section{Barry J. Huebert}

A

decade ago, the idea that oceanic phytoplankton regulate climate ${ }^{1}$ by producing dimethyl sulphide (DMS) led to the conventional wisdom that only natural DMS-derived sulphate aerosols influence cloud reflectivity (and thus the balance of radiation reaching the Earth) in remote marine areas. According to Capaldo et al. ${ }^{2}$ (on page 743 of this issue), there is another important sulphur source in the postindustrial era: sulphur dioxide pollution from ships burning fossil fuels. The global atmospheric sulphur model used by Capaldo et al., which is the first to incorporate a new inventory of emissions from worldwide shipping $^{3}$, not only tells us that the effect of human activity on aerosols and climate is greater than we previously thought, but also that attempts to fit earlier models to data may have been misleading. This isn't the first time air pollution has been poorly quantified, but it is worth investigating how the omission of ship exhausts may have led to misunderstandings in other aspects of atmospheric chemistry models.

Clouds reflect more sunlight back to space when they form on larger numbers of aerosol particles. It has been argued ${ }^{1}$ that more DMS might generate more sulphate aerosols and therefore create greater concentrations of cloud-condensation nuclei, which would make clouds brighter and cool the Earth a little (Fig. 1). Now we learn that shipping may generate more $\mathrm{SO}_{2}$ above parts of the oceans in the Northern Hemisphere than DMS does. The Capaldo et al. study ${ }^{2}$ is revealing in what it says about the confidence we should place in numerical models. Previous models neglected ships, but now that we have a reasonable inventory of ship emissions, the predictions are naturally quite different. This work represents a substantial change in our picture of atmospheric sulphur over much of the Earth. I think it's good for us to realize now and then that we haven't discovered everything of interest, and surprises are still out there.

We gain confidence in models as representations of reality when they predict spatial and temporal patterns that we can observe independently. Measurements on the scale of the phenomena the models are describing - are the sole means of

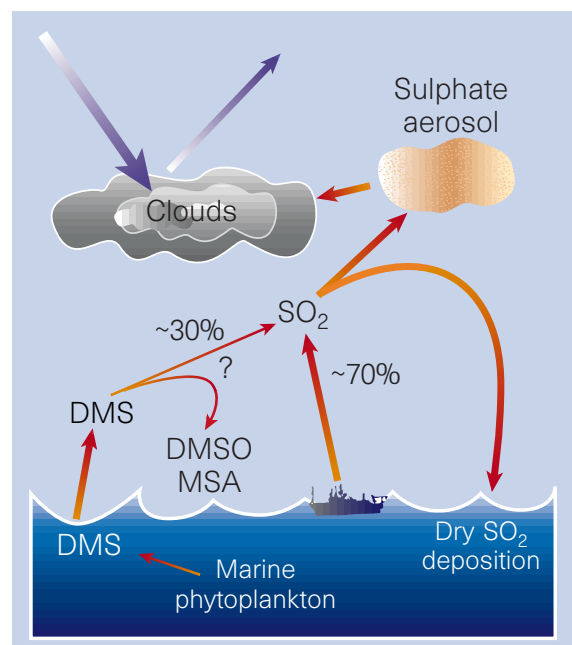

Figure 1 Sources and sinks of sulphur in the middle of the North Atlantic. Capaldo et al. ${ }^{2}$ show that ship emissions generate far more $\mathrm{SO}_{2}$ than previously assumed where there is heavy ship traffic. The main natural source of $\mathrm{SO}_{2}$ over the oceans comes from oxidation of dimethyl sulphide (DMS) produced by phytoplankton. Some of this $\mathrm{SO}_{2}$ will be oxidized to sulphate aerosols that affect the reflectivity of clouds, thereby cooling the Earth. Previous climate models did not consider ship emissions, and so had to derive all their $\mathrm{SO}_{2}$ from DMS, using estimates of net yields (? mols of $\mathrm{SO}_{2}$ produced per mol DMS consumed) of almost 1. Models with smaller net yields may now do a better job of matching observations, because much of the observed $\mathrm{SO}_{2}$ may be derived from ships. DMSO, dimethyl sulphoxide; MSA, methanesulphonic acid.

testing model predictions. Capaldo et al. ${ }^{2}$ make an effort to compare their model results with measured $\mathrm{SO}_{2}$ concentrations, but the results are not very satisfying. The model overpredicts most concentrations of $\mathrm{SO}_{2}$; the addition of ship emissions causes only a slight improvement. This may be due in part to the fact that we have very few remote $\mathrm{SO}_{2}$ measurements with which to constrain sulphur models: the need for high-quality time-series data on sulphur species at remote sites is acute. Clearly, we still have a long way to go.

Nonetheless, this new inventory may change some of the parameters used in mod- els of the pre- and post-industrial atmospheres. A good example is the branching of DMS products between $\mathrm{SO}_{2}$ (some of which might form sulphate particles) and a variety of other species, including dimethyl sulphoxide and methanesulphonic acid (Fig. 1 ). The net yield of $\mathrm{SO}_{2}$ is defined as the fraction of $\mathrm{SO}_{2}$ produced from oxidized DMS. Published net yields of $\mathrm{SO}_{2}$ from $\mathrm{DMS}^{4-6}$ range from 0.1 to almost 1 , so there is a lot of room for improvement. In fact, even models of pre-industrial sulphur chemistry may be changed by the new data on $\mathrm{SO}_{2}$ emissions from shipping, because observations of the present (polluted) atmosphere have been used to infer (possibly erroneous) net yields used in pre-industrial models.

It's not an easy matter to determine the net yield of $\mathrm{SO}_{2}$ : laboratory studies simply cannot mimic the wide range of conditions that ambient air experiences. To determine the net yield in the field, one must quantify not only the DMS source, but also the formation and loss rates of all related species. This has been achieved only in extremely clean environments, such as Antarctica ${ }^{7}$ and Christmas Island in the mid-Pacific ${ }^{8}$, where the system is sufficiently simple for one to constrain the important variables. (It remains to be seen, though, whether such pristine environments are good analogues of polluted ones.)

The alternative is to use the net yield of $\mathrm{SO}_{2}$ as an adjustable parameter ${ }^{9}:$ a knob that can be twiddled to make large-scale models fit observations of sulphur species. But, because poorly constrained loss processes and other $\mathrm{SO}_{2}$ sources (such as the shipping described by Capaldo et al. ${ }^{2}$ ) also affect the $\mathrm{SO}_{2}$ concentration, this approach is probably misleading. With the extra $\mathrm{SO}_{2}$ from shipping, modellers won't have to create all their $\mathrm{SO}_{2}$ from DMS, so a smaller net yield from DMS might produce sensible concentrations. Clearly, a lot of data will need to be reinterpreted in light of our new knowledge of sulphur emissions from ships.

We now have strong evidence that the 'unpolluted' part of the Earth is considerably smaller than we believed just a year ago. One wonders whether the impact of this newly quantified $\mathrm{SO}_{2}$ pollution extends beyond the observed reflective tracks that follow shipping routes in satellite images of clouds ${ }^{10}$. It is also evident that sulphur emissions from ships can equal or exceed land-based sources in coastal regions, affecting air quality in these areas. The work by Capaldo et al. provides the first good argument for reducing sulphur pollution from shipping.

It's not very comforting to know that our waste is affecting an even larger region. It is comforting, though, to see that the tools for making these assessments are getting sharper.

Barry J. Huebert is in the Department of 